\title{
Classification of Fused SAR/EO Images Using Transformation of Fusion Classification Class Label

\author{
Chul-Soo Ye
} \\ Department of Ubiquitous IT, Far East University
}

\begin{abstract}
Strong backscattering features from high-resolution Synthetic Aperture Rader (SAR) image provide useful information to analyze earth surface characteristics such as man-made objects in urban areas. The SAR image has, however, some limitations on description of detail information in urban areas compared to optical images. In this paper, we propose a new classification method using a fused SAR and Electro-Optical (EO) image, which provides more informative classification result than that of a single-sensor SAR image classification. The experimental results showed that the proposed method achieved successful results in combination of the SAR image classification and EO image characteristics.
\end{abstract}

Key Words : SAR-EO Image Fusion, Maximum Likelihood Classification, Initial Classification Class Label, Fusion Classification Class Label, Final Fusion Classification Label

\section{Introduction}

Multi-sensor image fusion provides additional information and improved analysis on target objects. Image fusion basically combines various information into a single fused information, which can offer a new way to analyze the target object. A variety of image fusion methods have been proposed (Li et al, 2003; Long and Gaungfang, 2007; Petrovic and Xydeas, 2004; Piella, 2003). Image fusion methods using SAR and EO images were also proposed in order to analyze urban areas (Fatone et al, 2001; Garzelli, 2002; Ye, 2009; Ye, 2010). Multiple fused images were obtained using SAR and EO images by a wavelet-based fusion of SAR and EO images using gradient and variance (Ye, 2010). This method can control the amount of each sensor image inserted into the fused image. This means we can obtain various types of fused images from SAR and EO images and also apply these fused images to some application fields such as classification, pattern recognition and etc.

In this paper, we propose the rules combining the multiple classification results of the fused images obtained from SAR and EO images into one classification result (Fig. 1). The advantage of the proposed method is that a new information (i.e.,

\footnotetext{
Received November 9, 2012; Revised December 14, 2012; Accepted December 17, 2012.

${ }^{\dagger}$ Corresponding Author: Chul-Soo Ye (csye@kdu.ac.kr)

This is an Open-Access article distributed under the terms of the Creative Commons Attribution Non-Commercial License (http://creativecommons.org/licenses/by-nc/3.0) which permits unrestricted non-commercial use, distribution, and reproduction in any medium, provided the original work is properly cited.
} 


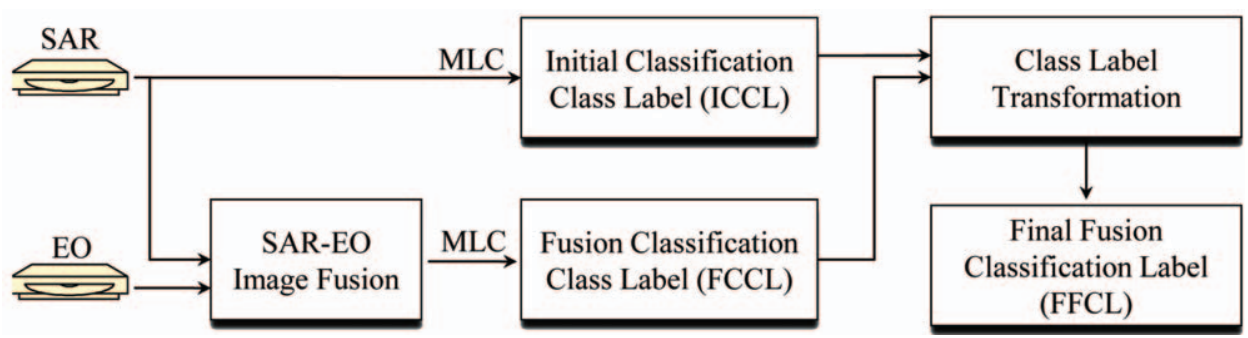

Fig. 1. Overall procedure of the proposed algorithm.

homogeneous intensity property) from EO image is inserted into the classification result of the fused image. We can obtain more informative land use classification result of SAR image, which is difficult to be obtained from SAR image alone. First, we create multiple fused images from SAR and EO images and then classify the pixels in each fused image into some class labels using Maximum Likelihood Classification (MLC) method. We combine the classified images into Fusion Classification Class Label (FCCL) using some combination rules. We also classify the SAR image into some class labels (Initial Classification Class Label, ICCL) using MLC method. The Final Fusion Classification Label (FFCL) is obtained by combining ICCL and FCCL.

\section{Methodology}

\section{1) Maximum Likelihood Classification (MLC)}

Maximum Likelihood Classification is a popular supervised classification method, where sample pixels are chosen to make training samples and then each pixel in the entire image is classified into the corresponding class based on the maximum likelihood. Denoting the number of the band images by $N$, the feature vector extracted from band image is defined as follows:

$$
\mathrm{x}=\left[x_{1}, x_{2}, \cdots, x_{N}\right]
$$

After selecting the training samples, the mean vector $\mathrm{m}_{\mathrm{k}}$ and the covariance matrix $\mathrm{C}_{\mathrm{k}}$ are computed as follows:

$$
\begin{gathered}
\mathrm{m}_{\mathrm{k}}=\frac{1}{M} \sum_{i=1}^{M} \mathrm{x}_{\mathrm{i}}^{\mathrm{k}} \\
\mathrm{C}_{\mathrm{k}}=\frac{1}{M} \sum_{i=1}^{M}\left(\mathrm{x}_{\mathrm{i}}^{\mathrm{k}}-m_{k}\right)\left(\mathrm{x}_{\mathrm{i}}^{\mathrm{k}}-m_{k}\right)^{T}
\end{gathered}
$$

whereis $M$ the total number of pixels in training set. The probability of each class is computed as follows: $p\left(\mathrm{x} \mid v_{k}\right)=\frac{1}{(2 \pi)^{\mathrm{N} / 2} \sqrt{\operatorname{det}(\mathrm{C})}} \exp \left(-\frac{1}{2}\left(\mathrm{x}-\mathrm{m}_{\mathrm{k}}\right)^{T} \mathrm{C}^{-1}\left(\mathrm{x}-\mathrm{m}_{\mathrm{k}}\right)\right)$ Finally we assign the class with maximum probability to the pixel as follows:

$$
v_{k}=\underset{v_{i}}{\operatorname{argmax}}\left(p\left(\mathrm{x} \mid v_{i}\right) p\left(v_{i}\right)\right),\left(k, i=1,2, \cdots, N_{C}\right)
$$

\section{2) Classification of SAR-EO fused image using MLC}

Fused image $I_{F}(i, j)$ generated from SAR image $I_{S}(i, j)$ and $\mathrm{EO}$ image $I_{O}(i, j)$ is defined as follows:

$$
\begin{gathered}
I_{k}^{F}(i, j)=W_{S}(k) \cdot I_{S}(i, j)+W_{O}(k) \cdot I_{O}(i, j), \\
W_{S}(k)+W_{O}(k)=1
\end{gathered}
$$

where $W_{S}(k)$ and $W_{S}(k)$ are fusion weights for SAR image and EO image, respectively. The fusion weights are determined by using the gradient and variance based fusion method (Ye, 2010). This fusion method employs the ratio of pixel intensity of SAR image over EO image, gradient magnitude and intensity variance. If the intensity value of SAR image is stronger than that of EO image, the fused image accepts the intensity value of SAR image as its intensity value. Otherwise the intensity value of the 


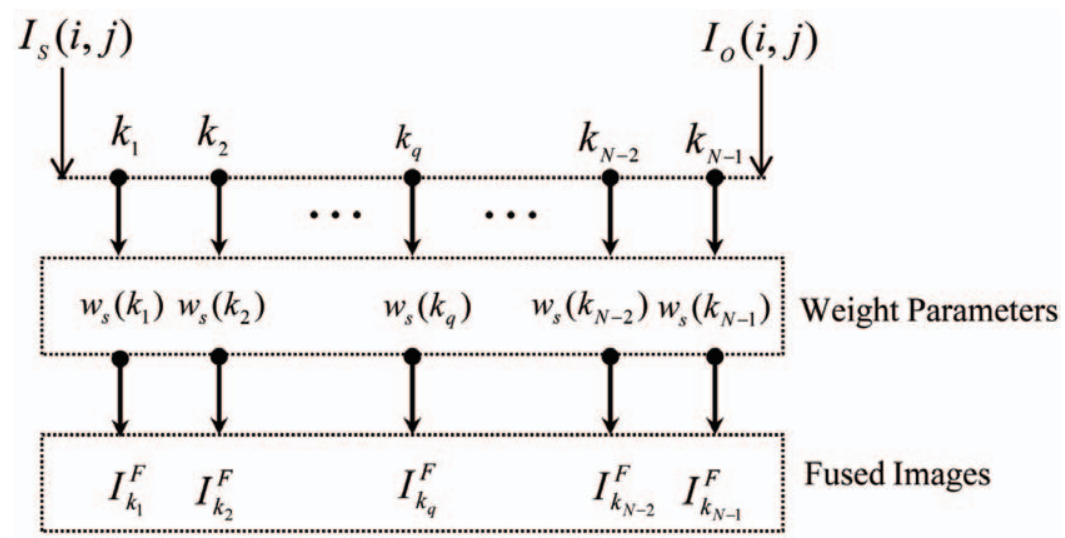

Fig. 2 Fused images according to discrete weight parameters.

fused image is determined by a weighted sum of SAR and EO images.

The weight value has two factors of the gradient magnitudes of pixels and the intensity variances of pixels in a local area in the two images as follows:

$$
\begin{gathered}
W_{S}(k)=k \cdot f_{S}\left(G_{S}, G_{O}, \sigma_{S}, \sigma_{O}\right) \\
W_{O}(k)=(1-k) \cdot f_{S}\left(G_{S}, G_{O}, \sigma_{S}, \sigma_{O}\right)
\end{gathered}
$$

where $G_{S}$ and $G_{O}$ are gradient magnitudes of the pixels of SAR image and EO image, respectively, and $\sigma_{S}$ and $\sigma_{O}$ are standard deviations of the intensity values of the pixels in a local area in SAR image and EO image, respectively. The parameter $k$ is a weight value controlled according to fusion purpose.

By controlling the parameter $k$ we can generate various fused images. For the parameter range $0<k<$ 1 , the discrete weight $k_{q}$ is given by

$$
k_{q}=\frac{1}{N} \cdot q,(q=1,2, \cdots, \mathrm{N}-1)
$$

For example, for $N=10$, the discrete weight $k_{q}$ has 9 weight values as follows:

$$
k_{q}=\left\{\frac{1}{10}, \frac{2}{10}, \frac{3}{10}, \frac{4}{10}, \frac{5}{10}, \frac{6}{10}, \frac{7}{10}, \frac{8}{10}, \frac{9}{10},\right\}
$$

Consequently, when the discrete weight $k_{q}$ is applied to equation (6), the fused image $I_{F}(i, j)$ is given by

$$
\begin{gathered}
I_{k_{q}}^{F}(i, j)=W_{S}\left(k_{q}\right) \cdot I_{S}(i, j)+W_{O}\left(k_{q}\right) \cdot I_{O}(i, j), \\
W_{S}\left(k_{q}\right)+W_{O}\left(k_{q}\right)=1
\end{gathered}
$$

Fig. 2 shows the relationship between the fused images and discrete weight parameters. We classify the fused image $I_{k_{q}}^{F}(i, j)$ by MLC method to generate the classified image $C_{k_{q}}^{F}(i, j)$ given by

$$
C_{k_{q}}^{F}(i, j)=\left\{c_{p} \mid P=1,2, \cdots, N_{C}\right\}
$$

where $N_{C}$ is the total number of classes and the element $c_{p}$ is a class label assigned to each class. In order to determine whether the classified image $C_{k_{q}}^{F}(i$, $j$ ) has class label $c_{p}$, class label detection function $N_{q}$, ${ }_{p}(i, j)$ is defined as follows:

$$
N_{q, p}(i, j)=N_{k_{q}, p}(i, j)\left[C_{k_{q}}^{F}(i, j)\right]=\left\{\begin{array}{l}
1 \text { if } C_{k_{q}}^{F}(i, j)=c_{p} \\
0 \quad \text { otherwise }
\end{array}\right.
$$

If the classified image $C_{k_{q}}^{F}(i, j)$ has class label $c_{p}$, the class label detection function is one, otherwise class label detection function is zero. The class label matrix element $C_{p}^{T}(i, j)$ is defined by

$$
C_{p}^{T}(i, j)=\sum_{i=1}^{N-1} N_{q, p}(i, j)
$$

The class label matrix element $C_{p}^{T}(i, j)$ represents the number of the classified images where the class label at $(i, j)$ has $c_{p}$. Table 1 shows the relationship between the class label detection function $N_{q, p}(i, j)$ and class label matrix element $C_{p}^{T}(i, j)$. For example, for class label $p=1$, if the classified image $C_{l}^{F}(i, j)$ has class label one, then the class label detection function $N_{1,1}(i, j)$ is one, otherwise $N_{1,1}(i, j)$ is zero. In this case, the class label matrix element $C_{l}^{T}(i, j)$ is 
Table 1. The relationship between the class label detection function $N_{q,}(i, \jmath)$ and class label matrix element $C_{p}^{T}(i, \jmath)$

\begin{tabular}{c|c|c|c|c|c}
\hline \hline Class & $C_{1}^{F}(i, j)$ & $C_{2}^{F}(i, j)$ & $\cdots$ & $C_{N-1}^{F}(i, j)$ & $C_{p}^{T}(i, j)$ \\
\hline 1 & $N_{1,1}(i, j)$ & $N_{2,1}(i, j)$ & $\cdots$ & $N_{N-1,1}(i, j)$ & $\sum_{q=1}^{N-1} N_{q, 1}(i, j)$ \\
\hline 2 & $N_{1,2}(i, j)$ & $N_{2,2}(i, j)$ & $\cdots$ & $N_{N-1,2}(i, j)$ & $\sum_{q=1}^{N-1} N_{q, 2}(i, j)$ \\
\hline$\vdots$ & & $\vdots$ & & & $\vdots$ \\
\hline 3 & $N_{1, N_{C}}(i, j)$ & $N_{2, N_{C}}(i, j)$ & $\cdots$ & $N_{N-1, N_{C}}(i, j)$ & $\sum_{q=1}^{N-1} N_{q, p}(i, j)$ \\
\hline \hline
\end{tabular}

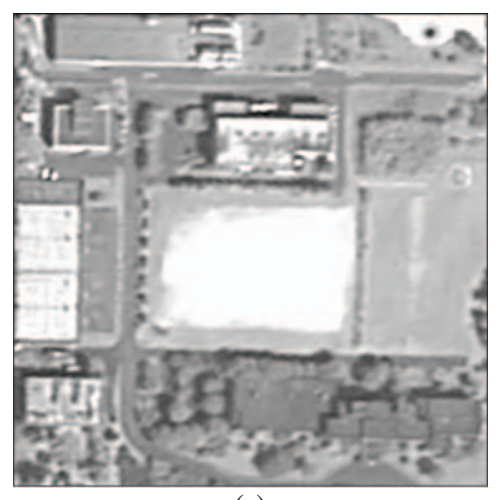

(a)

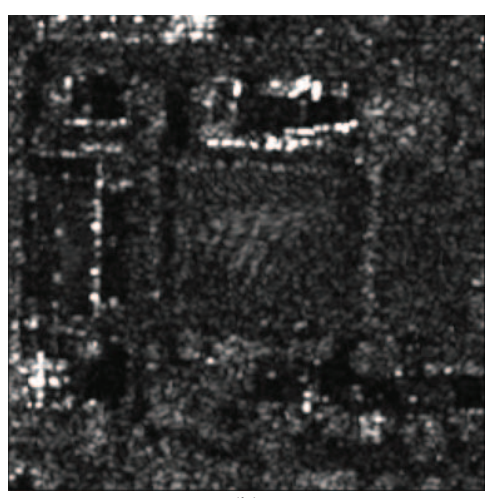

(b)

Fig. 3. Sample images (a) high intensity pixels in EO image (b) corresponding area in SAR image.

given by

$$
C_{l}^{T}(i, j)=\sum_{i=1}^{N-1} N_{q, 1}(i, j)=N_{1,1}(i, j)+N_{2,1}(i, j)
$$

This means the number of classified images where the class label at $(i, j)$ has one. After computing the class label matrix element $C_{p}^{T}(i, j)$, the class label $p_{F}$, which we call Fusion Classification Class Label (FCCL), is defined as the class label of the maximum class label matrix element $C_{p}^{T}(i, j)$ as follows:

$$
p_{F}=\underset{p}{\operatorname{argmax}}\left\{C_{p}^{T}(i, j)\right\}
$$

\section{3) Transformation of the Fusion Classification Class Label (FCCL)}

A fused SAR-EO image has mixed characteristics of SAR and EO images. If the parameter approaches zero, the amount of the SAR signal in the fused SAR/EO image increases. On the other hand, if the parameter approaches one, the amount of the EO signal in the fused SAR/EO image increases. If the intensity value of SAR image is weaker than that of EO image, the intensity value of the fused image is determined by a weighted sum of SAR and EO images. As shown in Fig. 3, those pixels that have high intensity value, low gradient magnitude and low variance are inserted to the fused image according to the fusion rules. In this case, the pixels with strong backscattering in SAR image and the high intensity pixels in homogeneous region in EO image will have same class in the fused image. Therefore we need to refer to the classification result of SAR image before we determine the final class of the fused image.

We define Initial Classification Class Label (ICCL) $C_{p}^{R}(i, j)$ obtained from classification of SAR image alone, Fusion Classification Class Label (FCCL) $C_{p}^{M}(i, j)$ and Final Fusion Classification Label (FFCL) $C_{p}^{L}(i, j)$ obtained from transformation 
Table 2. Three classes for TerraSAR-X satellite image

\begin{tabular}{c|c|c}
\hline \hline Class & Intensity & Land use types \\
\hline$c_{1}^{R}$ & High intensity pixels & Strong backscattering objects \\
\hline$c_{2}^{R}$ & Middle intensity pixels & Forset, grass, farmland, etc. \\
\hline$c_{3}^{R}$ & Low intensity pixels & Shadow, Water \\
\hline \hline
\end{tabular}

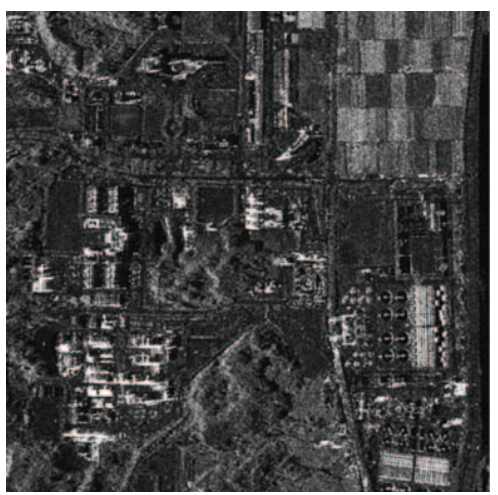

(a)

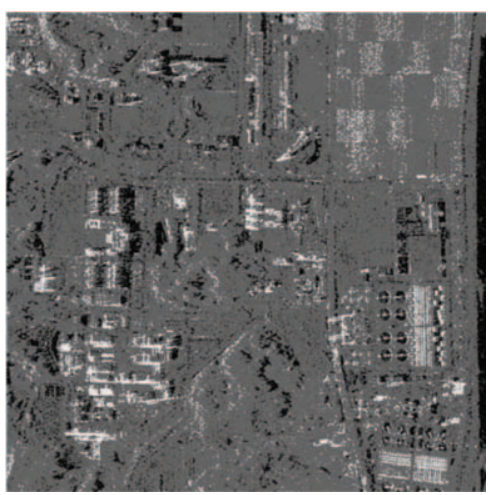

(b)

Fig. 4. Initial classification of TerraSAR-X satellite image (a) TerraSAR-X satellite image (b) Initial Classification Class Label (ICCL) image classified by MLC.

Table 3. Transformation of the Fusion Classification Class Label (FCCL) into the Final Fusion Classification Label (FFCL)

\begin{tabular}{c|c|l}
\hline \hline FCCL & FFCL & \multicolumn{1}{c}{ Conditions } \\
\hline \multirow{2}{*}{$c_{1}^{M}$} & $c_{1}^{M} \rightarrow c_{1}^{L}$ & $C_{p}^{R}(i, j) \neq c_{3}^{R}$ \\
& $c_{2}^{M} \rightarrow c_{2}^{L}$ & $C_{p}^{R}(i, j)=c_{2}^{R}$ \\
& $c_{3}^{M} \rightarrow c_{3}^{L}$ & $C_{p}^{R}(i, j)=c_{3}^{R}$ \\
\hline \multirow{2}{*}{$c_{2}^{M}$} & $c_{2}^{M} \rightarrow c_{2}^{L}$ & $C_{p}^{R}(i, j) \neq c_{3}^{R}$ \\
\cline { 2 - 3 } & & $C_{p}^{R}(i, j)=c_{3}^{R} \wedge C_{2}^{T}(i, j)>(N-1) / 2$ \\
\hline$c_{3}^{M}$ & $c_{3}^{M} \rightarrow c_{3}^{L}$ & $C_{p}^{R}(i, j)=c_{3}^{R}$ \\
\hline$c_{1}^{M}, c_{2}^{M}, c_{3}^{M}$ & new label $\rightarrow c_{4}^{L}$ & $C_{p}^{R}(i, j)=c_{3}^{R} \wedge C_{2}^{T}(i, j) \leq(N-1) / 2$ \\
\hline
\end{tabular}

of the FCCL are, respectively, as follows :

$$
\begin{aligned}
& C_{p}^{R}(i, j)=\left\{C_{p}^{R} \mid p=1,2, \cdots, N_{C}\right\} \\
& C_{p}^{M}(i, j)=\left\{C_{p}^{M} \mid p=1,2, \cdots, N_{C}\right\} \\
& C_{p}^{L}(i, j)=\left\{C_{p}^{L} \mid p=1,2, \cdots, N_{D}\right\}
\end{aligned}
$$

where $N_{C}$ and $N_{D}$ are the total number of the classes in ICCL and FCCL, respectively.

We assume that the total number of the classes in ICCL and FCCL for TerraSAR-X satellite image is three, respectively $\left(N_{C}=N_{D}=3\right)$. Table 2 shows the three classes for TerraSAR-X satellite image according to the level of pixel intensity. The land use types of TerraSAR-X satellite image such as strong backscattering object, shadow and water are easily discriminated based on the intensity of pixels. On the other hand, the land use type belonging to middle level intensity is difficult to be classified into more detail classes. Fig. 4 shows TerraSAR-X satellite image and ICCL image classified by MLC. We assigned pixel intensities 230, 100 and 0 to the class label $c_{1}^{R}, c_{2}^{R}$ and $c_{3}^{R}$ in the class label image, respectively.

The FCCL is determined as the class label with 
maximum class label matrix element and has three class labels $c_{1}^{M}, c_{2}^{M}$ and $c_{3}^{M}$, while the FFCL has four class labels $c_{1}^{L}, c_{2}^{L}, c_{3}^{L}$ and $c_{4}^{L}$, Table 3 shows the conditions for transformation of the FCCL into the FFCL.

\section{(1) Transformation of FCCL $C_{1}^{M}$}

If the initial classification class label (ICCL) $C_{p}^{R}(i$, j) satisfies the condition $C_{p}^{R}(i, j) \neq c_{3}^{R}$, then the fusion classification class label (FCCL) $c_{1}^{M}$ is assigned to the final fusion classification label (FFCL) $c_{1}^{L}$, that is, if the initial classification class label is not the class label $\left(c_{3}^{R}\right)$ belonging to low intensity pixels, then we assign $c_{1}^{M}$ to $c_{1}^{L}$. Although the fusion classification class label has the class label $c_{1}^{M}$, we assign $c_{2}^{M}$ and $c_{3}^{M}$ to $c_{2}^{L}$ and $c_{3}^{L}$, respectively. This means that if a pixel that is not classified into $c_{1}^{R}$ (that is, high intensity pixel) in initial classification class label image, we accept the classification result of the initial classification class label image (that is, $c_{2}^{M}=c_{2}^{R}$ and $c_{3}^{M}=c_{3}^{R}$ ) as the final fusion classification label $c_{2}^{L}$ and $c_{3}^{L}$, respectively. This condition is necessary for the pixels that have high intensity value, low gradient magnitude and low variance in the EO image, while the corresponding pixels in the SAR image do not have strong backscattering.

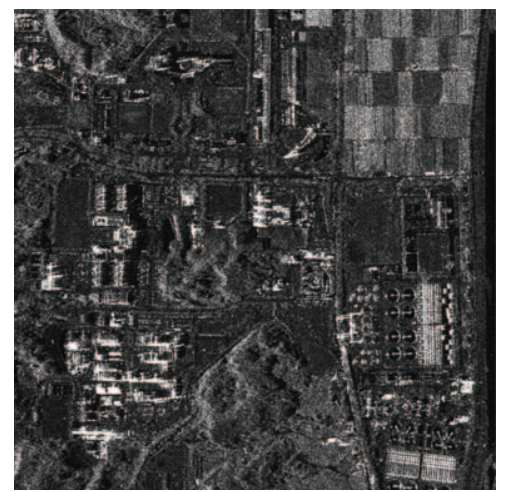

(a)

\section{(2) Transformation of FCCL $C_{2}^{M}$}

If the initial classification class label $C_{p}^{R}(i, j)$ satisfies the condition $C_{p}^{R}(i, j) \neq c_{3}^{R}$, then the fusion classification class label $c_{2}^{M}$ is assigned to the final fusion classification label $c_{2}^{L}$. According to the second condition $C_{p}^{R}(i, j)=c_{3}^{R} \wedge C_{2}^{T}(i, j)>(N-1) / 2$, if the initial classification class label $C_{p}^{R}(i, j)$ has the class label $c_{3}^{R}$ (that is, low intensity pixels) and the class label matrix element $C_{2}^{T}(i, j)$ is larger than a 50 percent of the total number of classification images as well, the fusion classification class label $c_{2}^{M}$ is assigned to the final fusion classification label $c_{2}^{L}$.

(3) Transformation of FCCL $C_{3}^{M}$ and creation of new class label $C_{4}^{L}$

If the initial classification class label $C_{p}^{R}(i, j)$ satisfies the condition $C_{p}^{R}(i, j) \neq c_{3}^{R}$, then the fusion classification class label is assigned to the final fusion classification label $c_{3}^{L}$. If the initial classification class label $C_{p}^{R}(i, j)$ has the class label $c_{3}^{R}$ (that is, low intensity pixels) and the class label matrix element $C_{2}^{T}(i, j)$ is smaller than a 50 percent of the total number of classification images as well, a new class label is assigned to the final fusion classification label $c_{4}^{L}$.

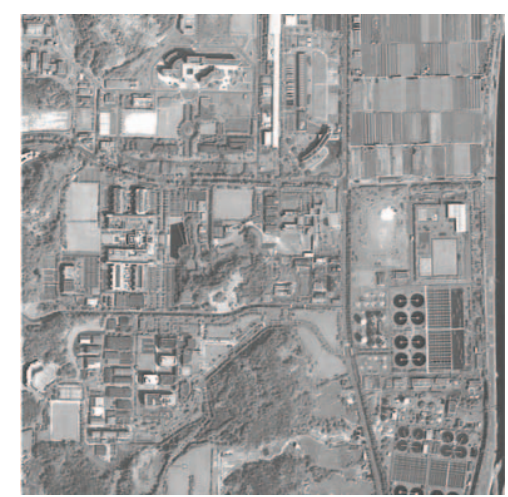

(b)

Fig. 5. Test images (a) a part of TerraSAR-X satellite imagery taken on July 11, 2008 (Daejeon) (b) the corresponding Kompsat-2 satellite imagery taken on September 13, 2008. 


\section{Experimental Results}

The proposed algorithm was tested using coregistered TerraSAR-X satellite image taken on July 11, 2008 and Kompsat-2 satellite image taken on September 13, 2008 (Fig.5). The TerraSAR-X satellite image has Geocoded Ellipsoid Corrected
(GEC) product type. The image-to-image registration of two images was carried out using affine transformation model. The average registration errors for five control points were 1.76 pixel for row direction, 0.57 pixel for column direction, respectively.

Fig. 6 shows the fusion results of TerraSAR-X satellite image and Kompsat-2 satellite image

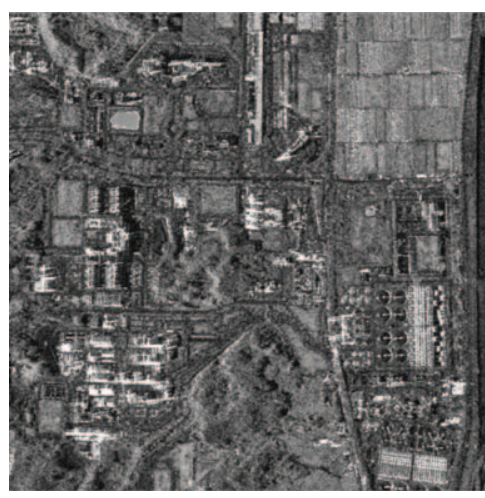

(a)

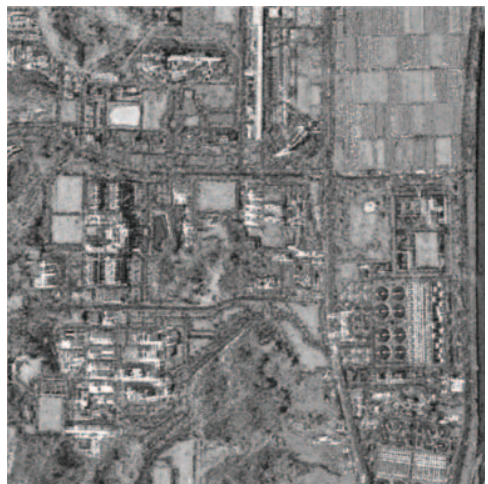

(d)

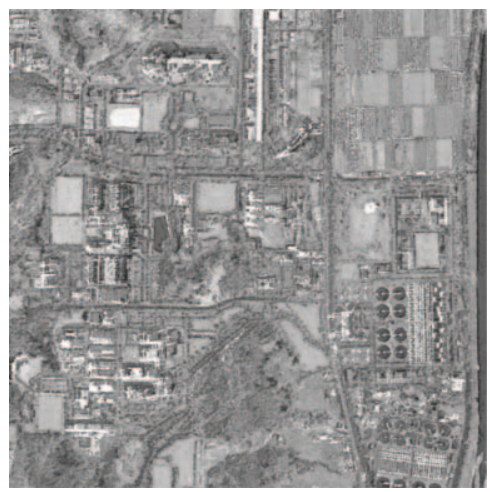

(g)

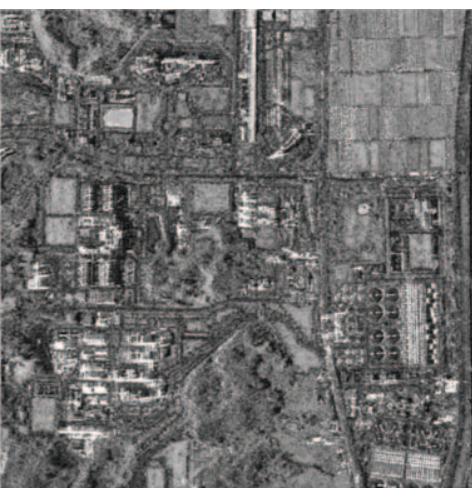

(b)

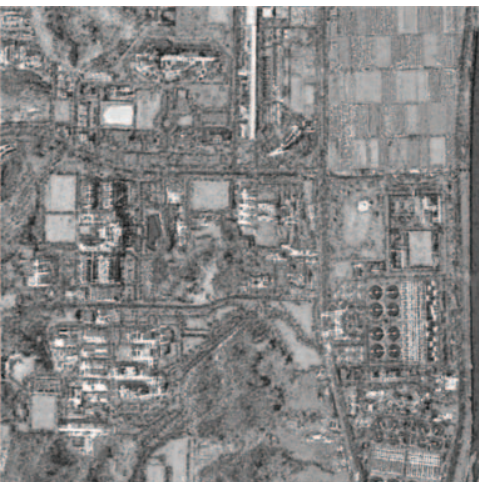

(e)

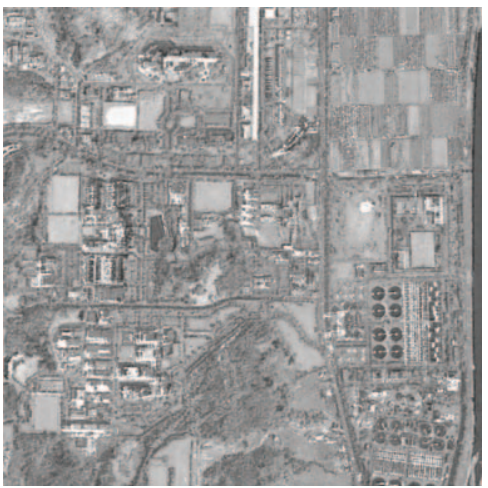

(h)

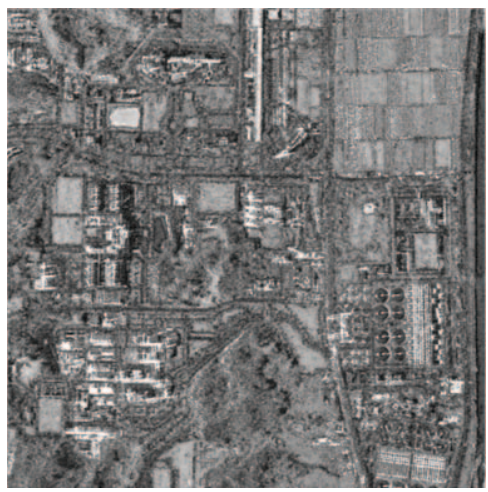

(c)

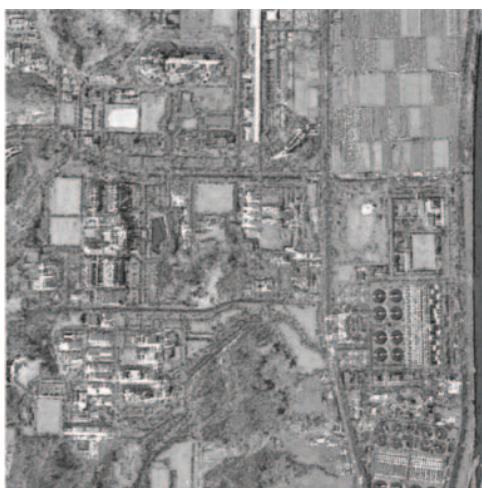

(f)

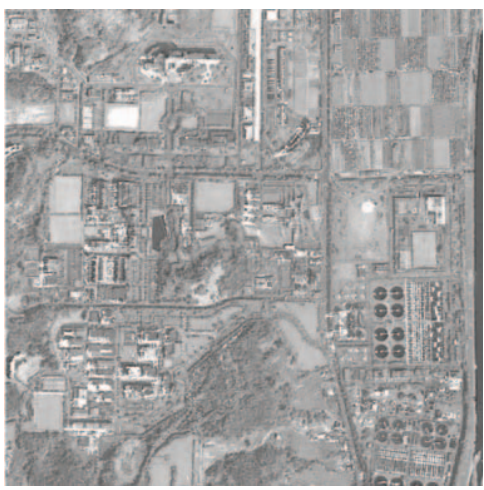

(i)

Fig. 6. The fusion results of TerraSAR-X satellite image and Kompsat-2 satellite image (a) $k_{q}=0.1$ (b) $k_{q}=0.2$ (c) $k_{q}=0.3$ (d) $k_{q}=$ 0.4 (e) $k_{q}=0.5$ (f) $k_{q}=0.6$ (g) $k_{q}=0.7$ (h) $k_{q}=0.8$ (i) $k_{q}=0.9$. 


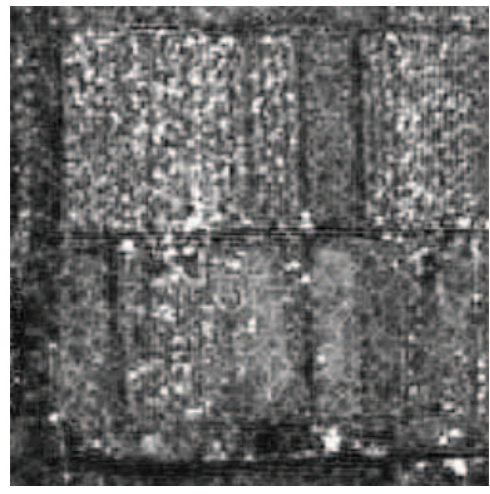

(a)

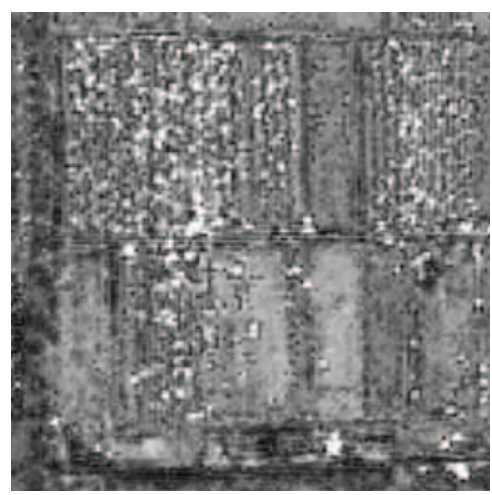

(d)

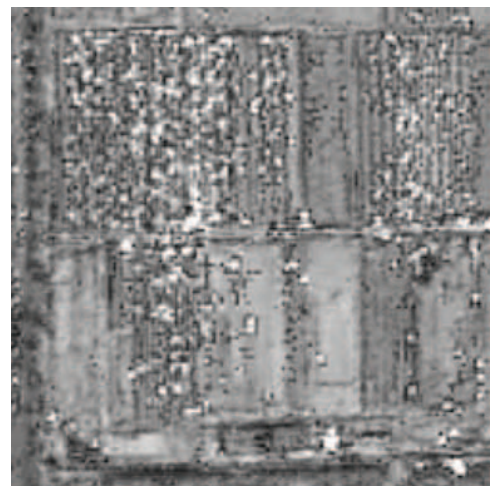

(g)

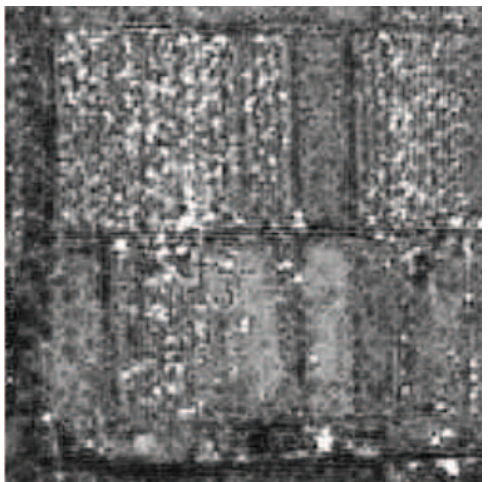

(b)

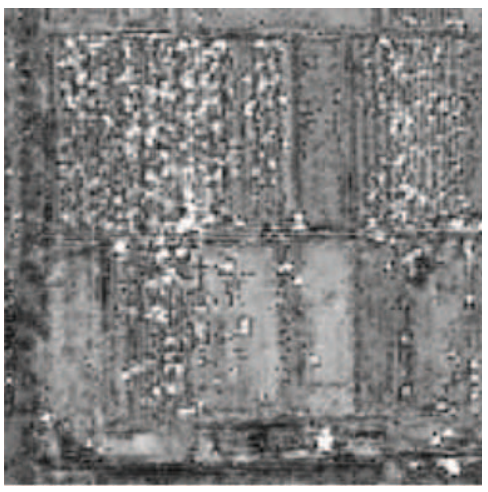

(e)

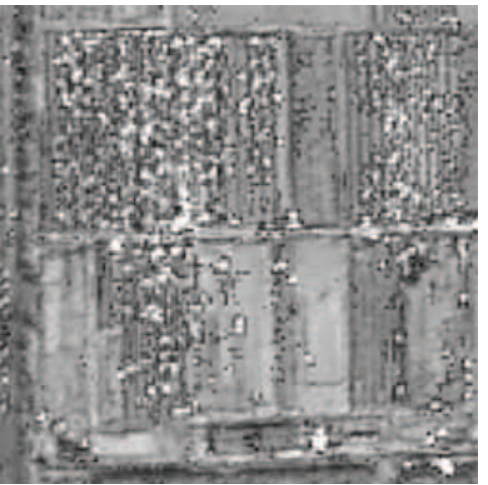

(h)

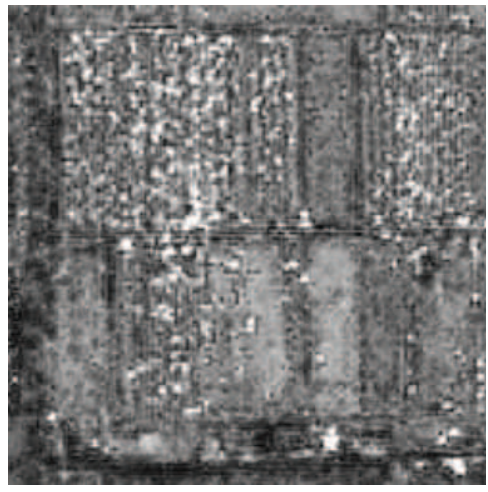

(c)

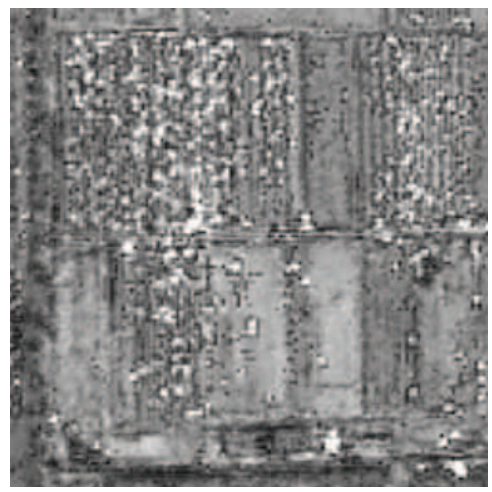

(f)

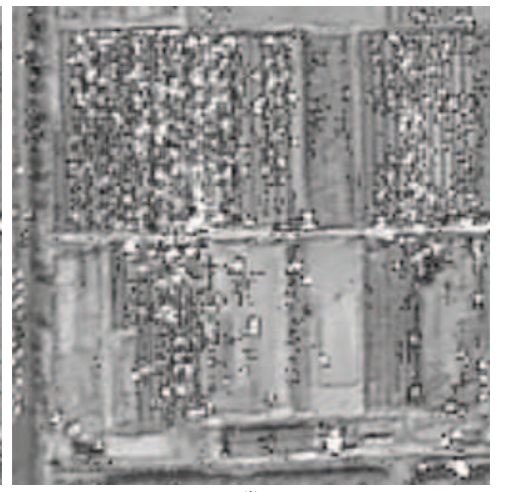

(i)

Fig. 7. Partial enlargement of the fusion results of Fig. 5 (a) $k_{q}=0.1$ (b) $k_{q}=0.2$ (c) $k_{q}=0.3$ (d) $k_{q}=0.4$ (e) $k_{q}=0.5$ (f) $k_{q}=0.6$ (g) $k_{q}$ $=0.7$ (h) $k_{q}=0.8$ (i) $k_{q}=0.9$.

according to the fusion weight $k_{q}$, which varies from 0.1 to 0.9 with the interval 0.1 . The amount of the pixels inserted from the Kompsat-2 satellite imagery in the fused SAR/EO image increases as $k_{q}$ increases. This is especially noticeable in the pixels in homogeneous intensity area of the Kompsat-2 satellite image as shown in Fig. 7.

Fig. 8 shows the classification result of the fused images in Fig. 5 by MLC. The total number of the classes in the FCCL image is three, the same as the total number of the classes in the ICCL. While the high intensity pixels belonging to the strong 


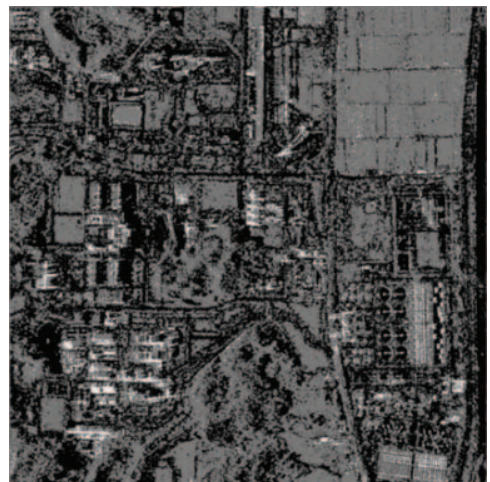

(a)

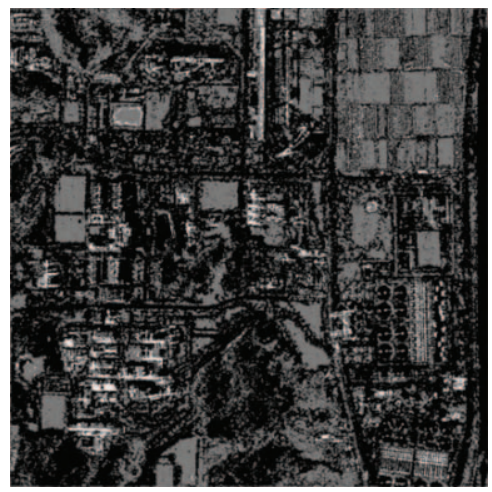

(d)

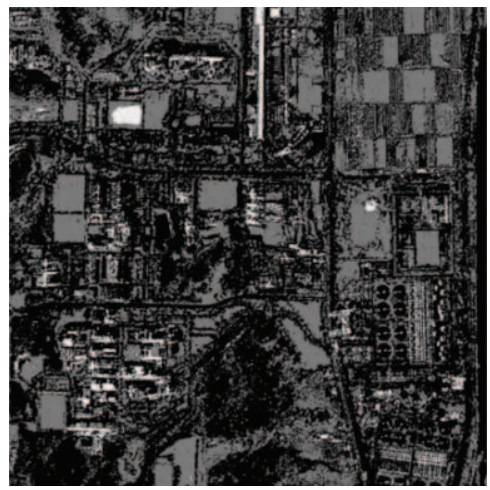

(g)

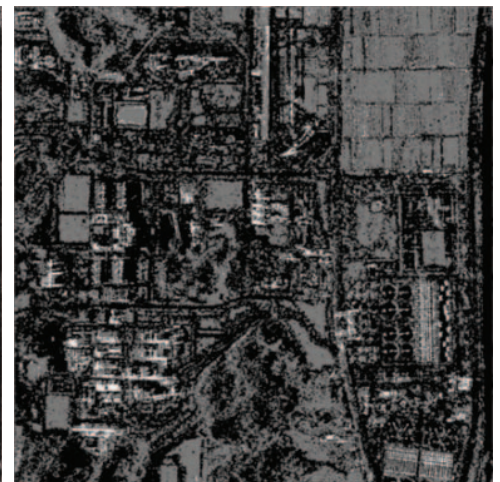

(b)

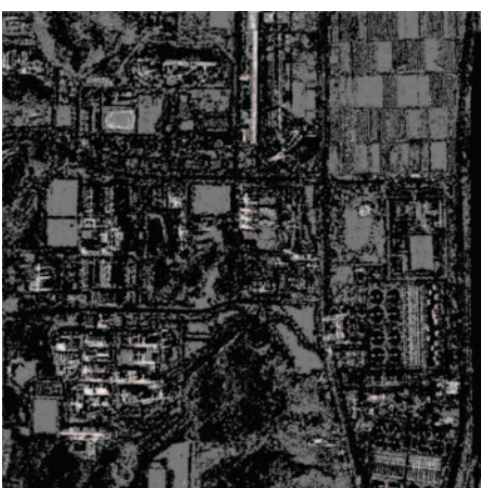

(e)

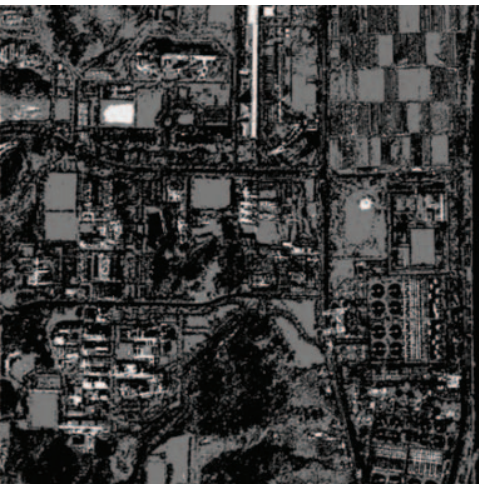

(h)

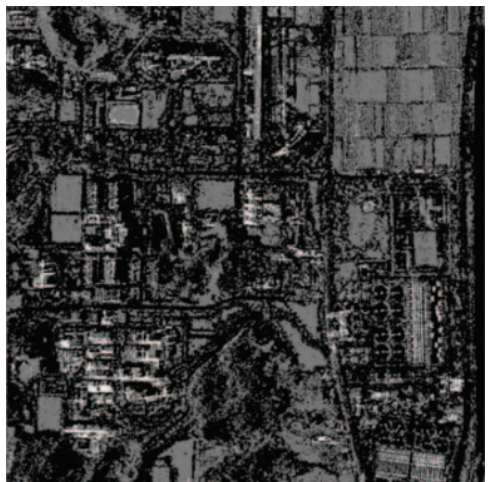

(c)

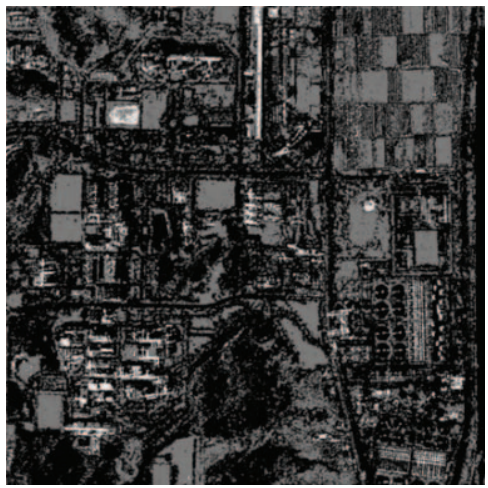

(f)

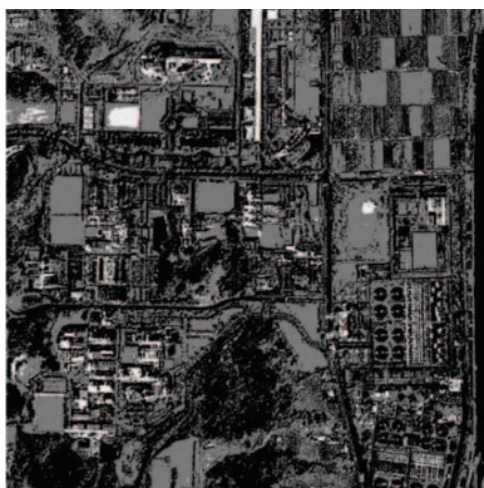

(i)

Fig. 8. Fusion Classification Class Label (FCCL) image. (a) $k_{q}=0.1$ (b) $k_{q}=0.2$ (c) $k_{q}=0.3$ (d) $k_{q}=0.4$ (e) $k_{q}=0.5$ (f) $k_{q}=0.6$ (g) $k_{q}$ $=0.7$ (h) $k_{q}=0.8$ (i) $k_{q}=0.9$.

backscattering in TerraSAR-X satellite image represents man-made objects, the high intensity pixels in Kompsat-2 satellite image are observed sometimes in the areas where man-made objects do not exist as shown in Fig. 9.

Table 4 shows the example of the transformation
Table 4. Transformation of the Fusion Classification Class Label (FCCL) $c_{2}^{M}$ into the Final Fusion Classification Label (FFCL) $c_{2}^{L}$

\begin{tabular}{c|c|c}
\hline \hline FCCL & Condition & FFCL \\
\hline \multirow{3}{*}{$c_{1}^{M}$} & $C_{p}^{R}(i, j) \neq c_{3}^{R}$ & $c_{1}^{M} \rightarrow c_{1}^{L}$ \\
\cline { 2 - 3 } & $C_{p}^{R}(i, j)=c_{2}^{R}$ & $c_{2}^{M} \rightarrow c_{2}^{L}$ \\
\cline { 2 - 3 } & $C_{p}^{R}(i, j)=c_{3}^{R}$ & $c_{3}^{M} \rightarrow c_{3}^{L}$ \\
\hline \hline
\end{tabular}




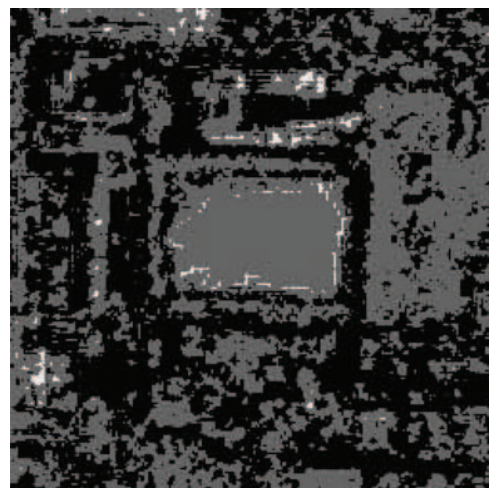

(a) $k_{q}=0.1$

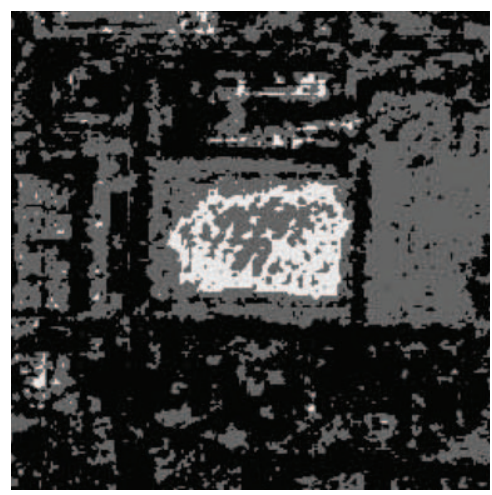

(d) $k_{q}=0.6$

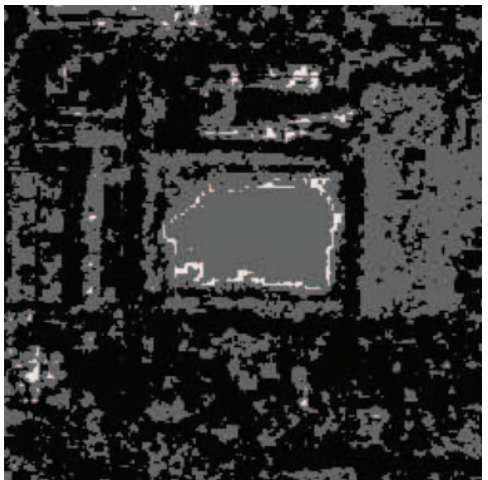

(b) $k_{q}=0.3$

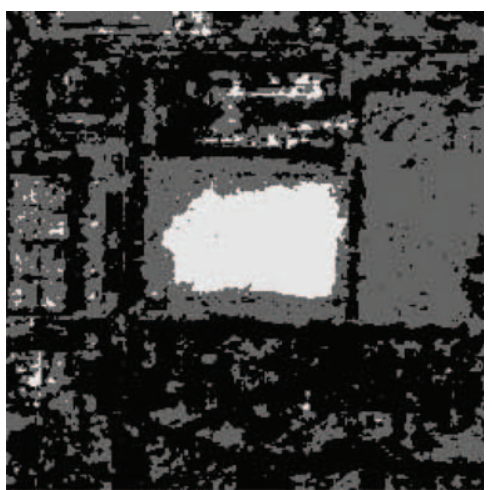

(e) $k_{q}=0.7$

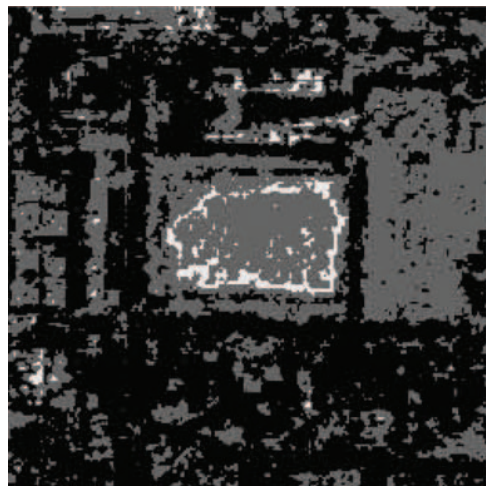

(c) $k_{q}=0.5$

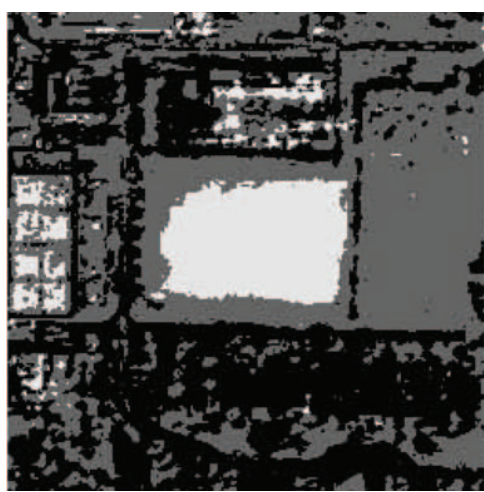

(f) $k_{q}=0.9$

Fig. 9. The change of the class labels in the fusion classification class label (FCCL) according to $k_{q}$.

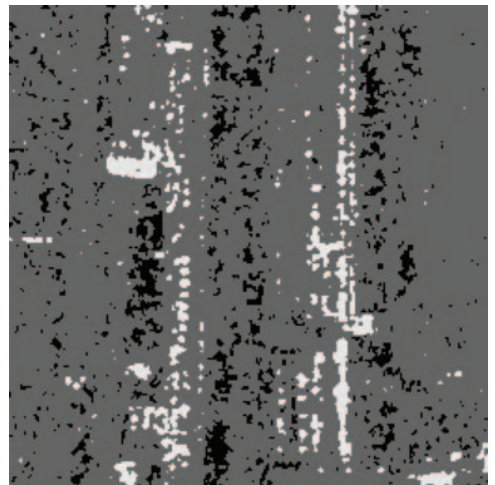

(a)

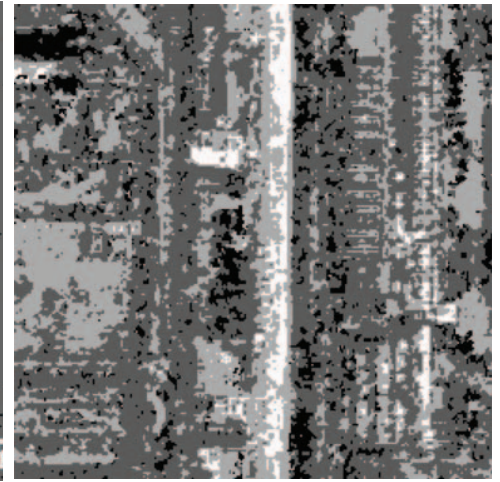

(b)

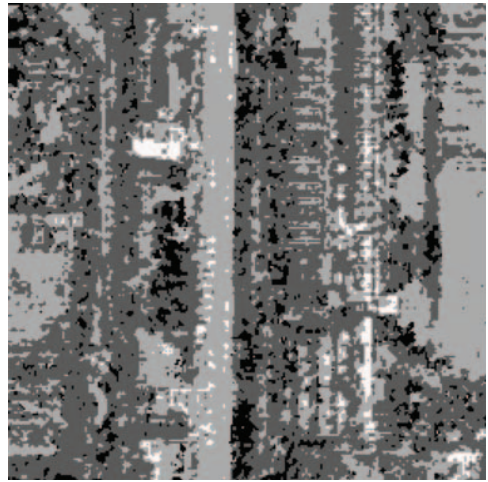

(c)

Fig. 10. Example of the class label transformation (a) ICCL (b) FCCL (c) FFCL.

of the Fusion Classification Class Label (FCCL) $c_{2}^{M}$. Though the FCCL has high intensity pixels $\left(c_{1}^{M}\right)$, the FCCL $c_{2}^{M}$ is assigned to the Final Fusion Classification Label (FFCL) when the condition $C_{p}^{R}(i, j)=c_{2}^{R}$ is satisfied. This means the FFCL accept the initial classification class label (medium intensity pixels) if the FFCL has high intensity pixels $\left(c_{1}^{M}\right)$ and ICCL, however, has the medium intensity pixel. Fig. 10 shows one example of the class label transformation. Because the high intensity pixels along the vertical 


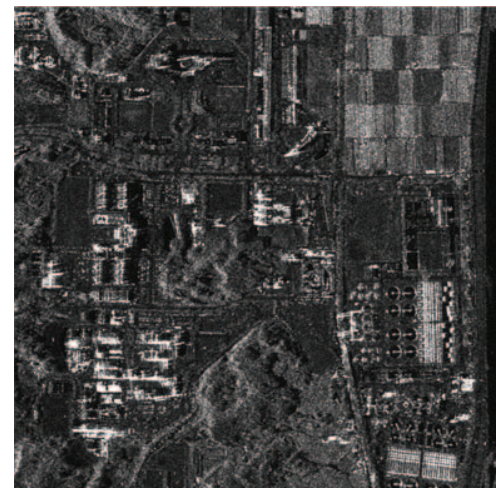

(a)

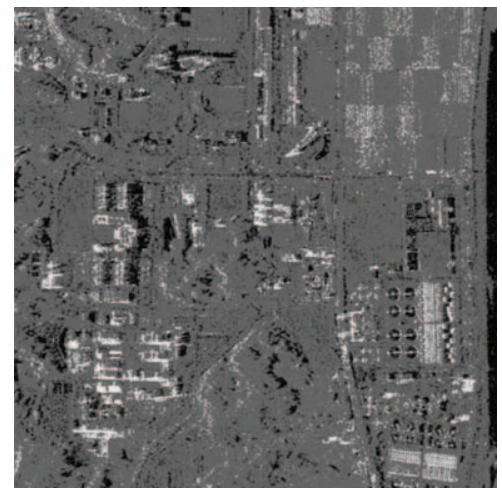

(c)

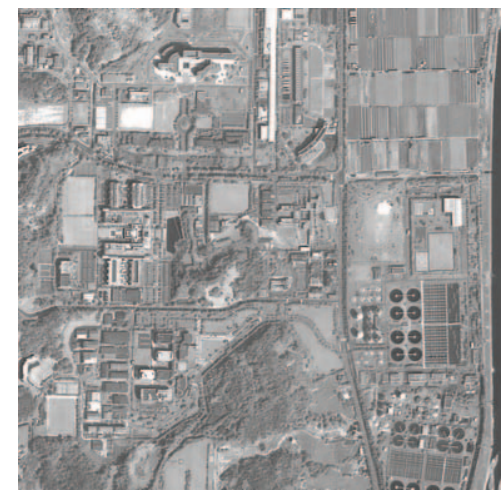

(b)

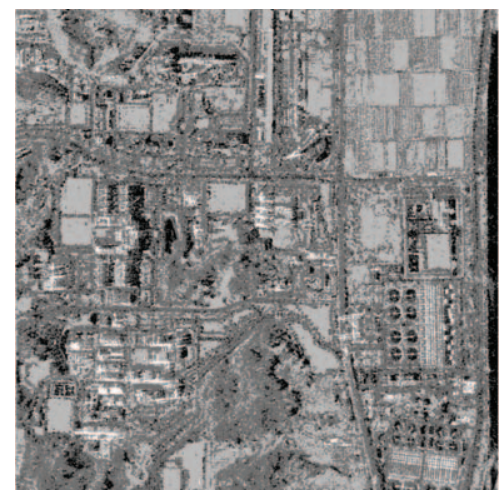

(d)

Fig. 11. Comparison of the classification results (a) TerraSAR-X satellite image (b) Kompsat-2 satellite image (c) ICCL image (d) FFCL image.

Table 5. Final Fusion Classification Label (FFCL) for the fused SAR/EO image

\begin{tabular}{c|l|l|c}
\hline \hline FFCL & \multicolumn{1}{|c|}{ Class Label Intensity } & \multicolumn{1}{|c}{ Land Property } & Intensity Level \\
\hline$c_{1}^{L}$ & High intensity & Strong backscattering region & 250 \\
\hline$c_{2}^{L}$ & Medium intensity type 1 & Homogeneous region & 180 \\
\hline$c_{3}^{L}$ & Medium intensity type 2 & Non-homogrnrous region & 90 \\
\hline$c_{4}^{L}$ & Low intensity & Shadow, water & 0 \\
\hline \hline
\end{tabular}

direction in the center of Fig. 10(b) have the class label $c_{1}^{M}$, while the initial classification class label $C_{p}^{R}(i, j)$ of those pixels has the class label $c_{1}^{R}$, the class label $c_{2}^{M}$ is assigned to the FFCL of those pixels as shown in Fig. 10(c).

The type of the final fusion classification label (FFCL) due to the transformation process in Table 3 is listed in Table 5. The total number of the FFCL is four, while the total number of the initial classification class labels and the fusion classification class labels is three. The pixels belonging to the class label $c_{2}^{R}$ (medium intensity pixels) in the initial classification class label are classified into the two medium intensity types, the medium intensity type 1 (homogeneous region) and the medium intensity type 2 (non-homogeneous region). Fig. 11(c) and Fig.11(d) show the ICCL image and the FFCL image for the fused TerraSAR-X/Kompsat-2 image, respectively. 


\section{Conclusions}

In this paper, we proposed the transformation rules combining the multiple classification results of the fused images into one classification result. The new definition of class label matrix element was proposed for the transformation of fusion classification class label (FCCL) to produce final fusion classification label (FFCL). Through the transformation of the FCCL into the FFCL, we created four final fusion classification labels for the fused SAR/EO image, which contained homogeneous intensity properties due to EO image. Future research concerning the combination of multiple classification will focus on fusing class labels concerning features of man-made objects in EO image with the final fusion classification label.

\section{Acknowledgements}

This study was supported by Korea Aerospace Research Institute through the program "Development Program of Korea Multi-Purpose SATellite-3 VII".

\section{References}

Fatone, L., P. Maponi, and F. Zirilli, 2001. Fusion of SAR/optical images to detect urban areas, Remote Sensing and Data Fusion over Urban Areas, IEEE/ISPRS Joint Workshop 2001, 217-221.
Garzelli, A., 2002. Wavelet-based fusion of optical and SAR image data over urban area, International Archives of Photogrammetry, Remote Sensing and Spatial Information Sciences 34(3): 59-62.

Li, Z., Z. Jing, G. Liu, S. Sun, and H. Leung, 2003. A region-based image fusion algorithm using multiresolution segmentation, IEEE Proceedings of 2003 Intelligent Transportation Systems, 96101.

Long, Z. and W. Guangfang, 2007. Study on adaptation of SAR/CCD image fusion algorithm, International Symposium on Photoelectronic Detection and Imaging 2007: Image Processing, Proceedings of SPIE, 6623: 66230.

Petrovic, V. and C. Xydeas, 2004. Gradient based multiresolution image fusion, IEEE Transactions on Image Processing, 4(2): 163-183.

Piella, G., 2003. A general framework for multiresolution image fusion: from pixels to regions, International Journal of Information Fusion, 4: 258-280.

Ye, C.S., 2009. Fusing optical and SAR images using wavelet transform and multiscale images, Proceedings of International Symposium on Remote Sensing 2009, Busan, Korea, Oct. 2830, 520-523.

Ye, C.S., 2010. Wavelet-based fusion of optical and radar image using gradient and variance, Korean Journal of Remote Sensing, 26(5): 581-591. 